\title{
The Mysterious Case of Missing Food from the Fridge: Sleep Eating on a Combination of Melatonin and Eszopiclone
}

\author{
Leilani Hernandez, $\mathrm{MPH}^{1^{*}}$ and Himanshu Agrawal, $\mathrm{MD}^{2}$ \\ ${ }^{1}$ Department of Medicine, Medical College of Wisconsin, USA \\ ${ }^{2}$ Department of Psychiatry and Behavioral Medicine, Medical College of Wisconsin, USA

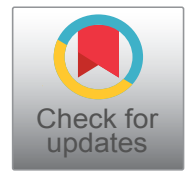

*Corresponding author: Leilani Hernandez, MPH, Department of Medicine, Medical College of Wisconsin, 8701 W, Watertown Plank Rd, Milwaukee, WI 53226, USA, Tel: (937)-241-6940

\begin{abstract}
Here we present a case of SRED induced by addition of melatonin to eszopiclone. For this patient, melatonin time release $3 \mathrm{mg}$ was added to eszopiclone $3 \mathrm{mg}$ at night for treatment of insomnia. He reported taking the melatonin for two nights and both mornings he found evidence that he had been up at night eating with no memory of it. He stopped the melatonin after these two nights and his sleep eating immediately resolved. Notably, he continued taking the eszopiclone after melatonin discontinuation. Prior research of SRED implicates a dopaminergic dysfunction in the pathogenesis of SRED. This case highlights a potential underlying mechanism for SRED involving a combination of melatonin and eszopiclone.
\end{abstract}

\section{Keywords}

Sleep related eating disorder, Melatonin, Eszopiclone, Obstructive sleep apnea

\section{Abbreviations}

CPAP: Continuous Positive Airway Pressure; GAD: General Anxiety Disorder; GERD: Gastroesophageal Reflux Disease; NREM: Non-Rapid Eye Movement; OSA: Obstructive Sleep Apnea; SRED: Sleep Related Eating Disorder

\section{Introduction}

Sleep related eating disorder (SRED) is a parasomnia associated with the non-rapid eye movement (NREM) phase of sleep, characterized by recurrent episodes of eating after sleep onset with full or partial amnesia of the episode [1,2]. SRED can result in adverse events such as weight gain and be potentially dangerous since patients have reported driving while asleep [1-4]. Known predisposing conditions of SRED include sleepwalking, restless leg syndrome, narcolepsy, obstructive sleep apnea (OSA), hypnotic medication use, and mental conditions such as major depressive disorder [2]. For patients with pre-disposing factors, pharmacologic treatments involve a combination of levodopa, bromocriptine, codeine, and clonazepam [2]. Dopaminergic agents are used as treatments since previous research indicate an underlying dopaminergic dysfunction for SRED.

Having said that, much remains unknown on what other factors can potentiate SRED. Here we present a case of SRED induced by addition of melatonin to eszopiclone and resolving immediately after the melatonin was stopped even though eszopiclone was continued.

\section{Report of Case}

A 69-year-old male who lives alone presented to clinic for medication management. Medical history was significant for generalized anxiety disorder (GAD), hypertension, hyperlipidemia, gastroesophageal reflux disease (GERD), and obstructive sleep apnea (OSA) treated with continuous positive airway pressure (CPAP). He is a former smoker and former user of alcohol and opioids. Last use for all of these substances was over 5 years ago. The patient had no prior history of parasomnias including sleepwalking, restless leg syndrome, narcolepsy, or periodic limb movement.

Since his prior visit, melatonin time release $3 \mathrm{mg}$ was added to eszopiclone $3 \mathrm{mg}$ at night for treatment of insomnia. Other medications included aspiring 81

Citation: Hernandez L, Agrawal H (2021) The Mysterious Case of Missing Food from the Fridge: Sleep Eating on a Combination of Melatonin and Eszopiclone. Int J Respir Pulm Med 8:154. doi. org/10.23937/2378-3516/1410154

Accepted: May 11, 2021; Published: May 13, 2021

Copyright: (C) 2021 Hernandez L, et al. This is an open-access article distributed under the terms of the Creative Commons Attribution License, which permits unrestricted use, distribution, and reproduction in any medium, provided the original author and source are credited 
$\mathrm{mg}$, atenolol $25 \mathrm{mg}$, gabapentin $300 \mathrm{mg}$ (1-2 tablets by mouth four times daily as needed), and ibuprofen 200 $\mathrm{mg}$ four times daily as needed for pain. The individual reported he did not take gabapentin at bedtime.

He reported taking the melatonin for two nights and both mornings he found evidence that he had been up at night eating. Clues included food missing from his refrigerator and food crumbs in his bed. He had no memory of eating during these two nights. He stopped the melatonin after these two nights and his sleep eating immediately resolved. Of note, this patient had no prior history of parasomnias including sleepwalking, restless leg syndrome, narcolepsy, and periodic limb movement.

\section{Discussion}

This patient developed SRED after the addition of time-released melatonin $3 \mathrm{mg}$ to eszopiclone $3 \mathrm{mg}$. Addition of melatonin may be a potential culprit for this patient's sudden onset of SRED. It started after the patient began taking both melatonin and eszopiclone and ceased immediately after melatonin discontinuation. To the authors' knowledge, there have been no reported cases of SRED on eszopiclone where as zolpidem is a hypnotic agent that has been notably implicated in sleep related disorders $[1,4,5]$. However, the patient did not report any parasomnias (including SRED) when he had been prescribed zolpidem in the past. One previous report discussed two patients with a history of sleep apnea who developed SRED after being switched to a different formulation of zolpidem [5].

Of note, these two cases using zolpidem were also using gabapentin [5]. This patient was also using gabapentin as needed for anxiety, however, categorically denies using gabapentin past early evening, and never at bedtime. This may warrant further investigation into whether gabapentin increases susceptibility to SRED.

Interestingly, Zapp, et al. reported on a patient with a history of chronic depression whose SRED resolved after the addition of extended-release melatonin [2].
These authors suggested for placebo-controlled studies to determine if melatonin can be used as a potential treatment for SRED. These cases potentially implicate melatonin in the pathogenesis for SRED as well. Future studies should be done to determine the potential underlying mechanism [6].

In summary, we report an unusual case of SRED induced with the addition of melatonin to eszopiclone in an elderly male with a complex medical history significant for depression, general anxiety disorder, and OSA. To our knowledge, this is the first case report to link melatonin and eszopiclone use with SRED. This case highlights a potential underlying mechanism for SRED involving a combination of melatonin and eszopiclone.

\section{Funding}

None.

\section{References}

1. Hoque R, Chesson AL Jr (2009) Zolpidem-induced sleepwalking, sleep related eating disorder, and sleep-driving: Fluorine-18-flourodeoxyglucose positron emission tomography analysis, and a literature review of other unexpected clinical effects of zolpidem. J Clin Sleep Med 5: 471-476.

2. Varghese $\mathrm{R}$, Rey de Castro J, Liendo $\mathrm{C}$, Schenck $\mathrm{CH}$ (2018) Two cases of sleep-related eating disorder responding promptly to low-dose sertraline therapy. J Clin Sleep Med 14: 1805-1808.

3. Zapp AA, Fischer EC, Deuschle M (2017) The effect of agomelatine and melatonin on sleep-related eating: $A$ case report. J Med Case Rep 11: 275.

4. Najjar M (2007) Zolpidem and amnestic sleep related eating disorder. J Clin Sleep Med 3: 637-638.

5. Chiang A, Krystal A (2008) Report of two cases where sleep related eating behavior occurred with the extended-release formulation but not the immediate-release formulation of a sedative-hypnotic agent. J Clin Sleep Med 4: 155-156.

6. Vetrugno R, Manconi M, Ferini-Strambi L, Provini F, Plazzi G, et al. (2006) Nocturnal eating: Sleep-related eating disorder or night eating syndrome? A videopolysomnographic study. Sleep 29: 949-954. 